\title{
Communicating transformation
}

\author{
COVID-19 has provided valuable insights into the role of logical-scientific communication during a crisis. Narrative \\ communication may be better suited for other public and planetary health challenges, including those that pertain \\ to food systems - and story-telling could be an effective agent for change.
}

S cience and politics have shared a remarkable stage - coalescing, colliding and sometimes socially distancing from each other - over the past year. COVID-19 briefings transmitted to our homes have laid bare mechanisms of science, operating boundaries of scientists and the necessity of political interpretation for handling multiple priorities. This has been fascinating to observe, and begs the question of whether lessons can be learned for other global challenges.

Can the public, for example, be persuaded to adopt healthy, sustainable food practices in the same way that they have been persuaded to wear masks, wash hands and socially distance? Would (and should) the public respond to the communication of risk in the same way?

Food crises seem to fall a little short on the dimensions of risk perception, described by Slovic, Fischhoff and others ${ }^{1}$, that would elicit the kind of mass behaviour change we have witnessed this year. The perception of risk is amplified in imposed situations (rather than voluntary ones), and situations where the behaviour of others has significant bearing on the outcome for an individual (where personal control is diminished) - and many challenges in public health nutrition and sustainable diets, for example, are associated with personal responsibility, voluntariness and a nudge towards the remedy. The familiar breeds less dread than the exotic - and food is certainly familiar. Where an adverse outcome is clear, imminent and potentially catastrophic, the perception of risk is greater than where a delay-effect exists for the outcome. The 'feeding 10 billion by 2050' message is used over and over - its familiarity, scale and distance may well reduce its potency. As Jonathan Safran Foer wrote in 2019, human alarm systems are not fit for conceptual threats, and we suffer "a fatigue of the imagination" for complex issues like climate change ${ }^{2}$ and food systems.

The citizen's toolkit with which to make food, lifestyle and climate-related decisions includes opinion, intuition, extrapolation, culture, worldview and experience. Within this space, particularly in this age of information and the echo chambers of social media, there is great potential for the entrenchment of views, fake news and 'truth decay' that are anathema to science. And yet, this is a sphere where science communicators for food-related behaviour change of individuals and food systems transformation within societies must operate. The terms of engagement, from information-provision to dialogue, must be renegotiated.

There is opportunity here with story-telling - a method that does not always sit well with scientific communities. Logical-scientific communication operates by trickled-down truths, context-free and holding across many scenarios. Narrative communication provides the context-specific root from which an individual can generalize upwards, with inductive reasoning ${ }^{3}$. While logical-scientific communication informs, narrative communication is intrinsically persuasive. Narrative communication is increasingly recognized as having potential in the communication of science on public health issues $^{4}$, and perhaps has a role for food systems transformation. Food is, after all, intensely political, intensely personal and makes for great story-telling - which has been highlighted by organizations such as Food Tank, EAT and the Global Food Security programme.

The prominent media coverage of 'the science' during the COVID-19 pandemic has brought attention to how science is communicated, and the role (and boundaries) of the scientist in informing, persuading and decision-making. With ethical tensions between the goals of informing and persuasion, the question also arises of whether the scientist and the story teller should be one and the same; the role of the advisor - the bridge between the scientific and political communities becomes evident here. Many of the public and planetary health challenges that relate to food are long in the making, complex and involve a multiplicity of stakeholders with a multiplicity of truths. While logical-scientific communication supports the urgency of food system transformation, expert story-telling may well be a more powerful tool for effecting behaviour change so that individuals and society follow the science.

Published online: 11 December 2020 https://doi.org/10.1038/s43016-020-00209-1

\footnotetext{
References

1. Schmidt, M. Investigating Risk Perception: A Short Introduction (2004); http://www.markusschmidt.eu/pdf/Intro_risk perception_Schmidt.pdf

2. Safran Foer, J. We are the Weather: Saving the Planet Begins at Breakfast (Hamish Hamilton, 2019).

3. Dahlstrom, M. F. Proc. Natl Acad. Sci. USA 111, (Suppl. 4) 13614-13620 (2014).

4. McCall, B., Shallcross, L., Wilson, M., Fuller, C. \& Hayward, A. BMJ Open 9, e030597 (2019).
} 